Gefässchirurgie $2015 \cdot 20: 287-288$

DOI 10.1007/s00772-015-0031-8

Online publiziert: 29. Mai 2015

(c) Springer-Verlag Berlin Heidelberg 2015

\author{
W. Hacke ${ }^{1} \cdot$ O. Jansen ${ }^{2} \cdot$ P. Ringleb ${ }^{1} \cdot$ H.-H. Eckstein ${ }^{3}$, für das SPACE-2 Steering \\ Committee \\ ${ }^{1}$ Neurologische Universitätsklinik Heidelberg, Heidelberg, Deutschland \\ ${ }^{2}$ Klinik für Radiologie und Neuroradiologie, Universitätsklinikum Kiel, Kiel, Deutschland \\ ${ }^{3}$ Klinik für Vaskuläre und Endovaskuläre Chirurgie, Klinikum rechts der Isar, \\ Technische Universität München, München, Deutschland
}

\title{
Behandlung asymptomatischer Karotisstenosen
}

\section{Eine verpasste Chance}

Die optimale Behandlung asymptomatischer arteriosklerotischer Abgangsstenosen der A. carotis interna ist umstritten [1, 2]. Ältere Studien aus den 1990er Jahren belegten einen geringen Vorteil der Karotisendarteriektomie (CEA) gegenüber der damals üblichen medikamentösen Prävention [3], aber es ist unklar, ob dies auf die Stentangioplastie („, carotid artery stenting" bzw. CAS) übertragen werden kann [4]. Ebenfalls offen bleibt die Frage, ob die Fortschritte in der medikamentösen Prävention (Stichworte: Statine und moderne Antihypertensiva) das Schlaganfallrisiko bei Karotisstenose so weit herabsetzen, dass eine Intervention (gleich ob CEA oder CAS) überhaupt noch einen präventiven Effekt hat [5].

Die SPACE-2-Studie ist eine von der Deutschen Forschungsgemeinschaft (DFG) im Rahmen des Programms „Klinische Studien" geförderte dreiarmige Studie (Best mögliche medikamentöse Therapie vs. CEA vs. CAS, Randomisierung 1:2:2, ISRCTN 78592017), bei der zunächst überprüft werden sollte, ob eine konsequente konservative Therapie einer invasiven Therapie hinsichtlich der Prävention von Schlaganfällen gleichwertig ist. Sollte die invasive Therapie überlegen sein, wäre als sekundäre Analyse der Vergleich zwischen CEA und Stenting gefolgt [6]. Aufgrund der relativ geringen zu erwartenden Schlaganfallrate wurde diese Studie für mehr als 3000 Patienten und eine Mindestbeobachtungszeit von fünf Jahren geplant.

\section{Unzureichende Rekrutierung}

Aufgrund der zu langsamen Rekrutierung und der Schwierigkeit, an allen Studienzentren alle drei Therapieoptionen mit den nötigen Qualitätsvoraussetzungen vorzuhalten, wurde mit Zustimmung der DFG das dreiarmige Studiendesign in zwei parallel laufende Studien mit jeweils 1:1 Randomisierung (konservativ vs. CEA und konservativ vs. CAS) geändert $[7,8]$. Dieses Studiendesign wird auch in der jetzt beginnenden vom National Institute of Health (NIH) geförderten amerikanischen Partnerstudie CREST-2 eingesetzt, die sich eng mit der SPACE-Gruppe über das Studiendesign und geplante gemeinsame Analysen abgestimmt hatte.

Leider musste die SPACE-2-Studie nach fast fünf Jahren Studiendauer auf Empfehlung der DFG gestoppt werden, da auch die Änderung des Studiendesigns nicht zu der notwendigen Steigerung der Einschlusszahlen geführt hatte. Bis zum Studienabbruch waren trotz ständiger Versuche, die Rekrutierungszahlen zu steigern nur 503 Patienten in 36 aktiven Studienzentren eingeschlossen worden.

\section{Viele Patienten kämen in Frage}

Von der DFG wurde bestätigt, dass die Fragestellung von SPACE-2 extrem wichtig sei, da geklärt werden muss, ob bei Patienten mit höhergradigen asymptomatischen Karotisstenosen eine konsequente konservative Therapie CAS oder CEA ersetzen kann und, falls nicht, CAS der CEA nicht unterlegen ist. Es gibt auch ausreichend viele Patienten: Immerhin wurden 2013 in Deutschland 12.096 Patienten mit einer asymptomatischen Karotisstenose operiert und weitere 2.452 mit einem Stent behandelt. Auch in den Studienzentren werden genügend Patienten gesehen, die für diese Studie in Frage kämen. Die drei Zentren mit den höchsten Patienteneinschlüssen haben zwischen 50 und 100 Patienten eingeschlossen. Wären diese Rekrutierungszahlen die Regel gewesen, müsste hier nicht über den Abbruch der Studie berichtet werden.

Ein wichtiger Grund dafür, dass für SPACE-2 - trotz vieler Patienten - dennoch so schlecht rekrutiert wurde, besteht darin, dass hierzulande viele potenzielle Studienpatienten mit dem Zufallsbefund einer asymptomatischen Karotisstenose in operative oder interventionelle Abteilungen eingewiesen werden. Gefäßchirurgen, (Neuro)-Radiologen und Kardiologen bestätigen, dass es sehr schwer ist, Patienten, die schon auf eine bestimmte Intervention gebahnt sind, davon zu überzeugen, besser an einer randomisierten Studie teilzunehmen, bei der es auch einen konservativen Arm gibt. Auch wird befürchtet, Zuweiser zu verärgern, wenn man nicht den erbetenen Eingriff durchführt, sondern dem Patienten erläutert, dass eine klinische Studie erst die Wirksamkeit der angeforderten Therapie beweisen müsse. Im Einzelfall könnten auch

Dieser Beitrag wurde erstpubliziert in Deutsches Ärzteblatt 2015: 112(7):A-272/B-234/C-230 
finanzielle Aspekte ausschlaggebend gewesen sein.

Immerhin werden im DRG-System Operationen und kathetergestützte Interventionen an der Halsschlagader mit einem Betrag von circa $6.000 €$ vergütet. Da inzwischen bei allen Kliniken inkl. der Universitätskliniken die wirtschaftlichen Zwänge erheblich sind, werden gerade von den operativen und interventionell tätigen Abteilungen jährliche Leistungssteigerungen erwartet. Studienpatienten die in den konservativen Behandlungsarm randomisiert wurden, wären für die Leistungsstatistik der invasiven Therapeuten und somit auch für das Krankenhausbudget verloren.

\section{Fehlende Anreize für Studie}

Dass dies auch ganz anders gehen kann, beweist die gerade veröffentlichte niederländische MR-CLEAN-Studie zur endovaskulären mechanischen Thrombektomie bei akutem Schlaganfall. In Holland wurde die interventionelle Therapie der Thrombektomie nur dann bezahlt, wenn sie im Rahmen dieser randomisierten Studie durchgeführt wurde. Das Ergebnis war, dass innerhalb von nur drei Jahren 500 Patienten in diese randomisierte Studie eingeschlossen werden konnten und dadurch die evidenzbasierte Überlegenheit der Thrombektomie nachgewiesen wurde [9]. In den USA wird die kathetergestützte Therapie von asymptomatischen Karotisstenosen derzeit nur bei Teilnahme an der CREST-2-Studie vergütet [10]. Ein derartiger Anreiz hätte ganz sicher auch an deutschen akademischen Zentren zu einer ausreichend schnellen Rekrutierung für die SPACE-2Studie geführt.

Das Scheitern von SPACE-2 bedeutet auch einen Rückschlag für die Kultur akademischer klinischer Studien und die Implementierung einer evidenzbasierten Medizin in Deutschland. Dies ist mehr als bedauerlich, da gerade in den letzten Jahren einige deutsche neurovaskuläre Studien international hoch beachtet publiziert wurden und aufgrund ihrer klaren Ergebnisse in entsprechende Leitlinien Einzug genommen haben. So wurde z. B. SPACE-1, trotz minimaler finanzieller Förderung, als erste von vier gro-
Ben internationalen randomisierten Studien zum Vergleich von CEA und CAS bei symptomatischen Karotisstenosen in LANCET publiziert [11]. Weitere Beispiele sind DESTINY [12] und die ebenfalls DFG-geförderte DESTINY-II-Studie [13], die dazu geführt haben, die Hemikraniektomie als lebensrettende Maßnahme bei raumforderndem Mediainfarkt zu etablieren.

\section{Umdenken ist notwendig}

Das traurige Ende von SPACE-2 ist somit auch ein Hinweis für die fehlende Fähigkeit großer deutscher Zentren, akademische Studien ohne finanziellen Anreiz erfolgreich zu gestalten, besonders wenn es um Medizinprodukte oder Interventionen geht, die trotz ausstehendem Beweis ihrer Wirksamkeit in unserem Gesundheitssystem großzügig bezahlt werden. Klinische Studien scheinen nur dann attraktiv, wenn die Industrie teuer dafür bezahlt. Wenn das, wie bei Medizinprodukten, nicht nötig ist, dann ist die Motivation gering.

Wir sollten aufhören, nach außen nach wissenschaftlichen Beweisen zu rufen, wenn wir nach innen nicht bereit, willens und möglicherweise auch nicht fähig sind, diese Beweise durch methodisch hochwertige saubere Studien zu erbringen. Darüber hinaus ist eine Änderung der Rahmenbedingungen notwendig. Klinische Forschung mit ausreichend großen $\mathrm{Pa}$ tientenkollektiven kann nicht funktionieren, wenn ökonomische Fehlanreize und ein unzureichendes akademisches Selbstverständnis, die Wahl einer möglicherweise unzureichend abgesicherten Therapie bestimmen. So gesehen sind auch die in dem aktuell diskutierten GKV-Versorgungsstärkungsgesetz vorgesehenen Erprobungsrichtlinien für Medizinprodukte der Klasse IIB und III ein Schritt in die richtige Richtung.

\section{Korrespondenzadresse}

\section{Univ. Prof. Dr. H.-H. Eckstein}

Klinik für Vaskuläre und Endovaskuläre

Chirurgie, Klinikum rechts der Isar

Technische Universität München

Ismaninger Str. 22, 81675 München

hheckstein@web.de
Einhaltung ethischer Richtlinien

Interessenkonflikt. W. Hacke, O. Jansen, P. Ringleb und $\mathrm{H}$.-H. Eckstein geben an, dass kein Interessenkonflikt besteht.

Dieser Beitrag beinhaltet keine Studien an Menschen oder Tieren.

\section{Literatur}

1. Eckstein $\mathrm{HH}$, Kühnl A et al (2013) Clinical practice guideline: the diagnosis, treatment and follow-up of extracranial carotid stenosis - a multidisciplinary German-Austrian guideline based on evidence and consensus. Deutsches Ärzteblatt Int 110:468476

2. Ringleb P, Berkefeld J, Eckstein HH (2012) S 3-Leitlinie extracranielle Carotisstenose; Kapitel 8.2: Wann und zu welchem Zeitpunkt besteht die Indikation zur Operation oder zur Intervention einer asymptomatischen/ symptomatischen Stenose? Gefässchirurgie 17:522-542

3. Halliday A, Harrison M et al 10-year stroke prevention after successful carotid endarterectomy for asymptomatic stenosis (ACST-1): a multicentre randomised trial. Lancet 2010;376:1074-1084

4. Bulbulia R, Halliday. ACST-2-an update (2013) A large, simple randomised trial to compare carotid endarterectomy versus carotid artery stenting to prevent stroke in asymptomatic patients. Gefässchirurgie 18:626-632

5. Kennedy F, Featherstone RL, Brown MM (2013) Current status of ECST-2. Gefässchirurgie 18:633637

6. Reiff T, Stingele R et al (2009) Stent-protected angioplasty in asymptomatic carotid artery stenosis vs. endarterectomy: SPACE2- a three-arm randomised-controlled clinical trial. Int J Stroke 4:294299

7. Reiff T, Eckstein HH et al (2013) Therapie asymptomatischer Karotisstenosen. Gefässchirurgie 18:621-625

8. Reiff T, Eckstein HH et al (2014) Modification of SPACE-2 study design. Int J Stroke 9:E12-13

9. Berkhemer OA, Fransen PSS et al (2014) A randomized trial of intraarterial treatment for acute ischemic stroke. NEJM 371:11-20

10. http://www.cms.gov/Medicare/Medicare-GeneralInformation/Medicare ApprovedFacilitie/CarotidArtery-Stenting-CAS-Investigational-Studies.html

11. Ringleb PA, Allenberg JR et al (2006) 30 day results from the SPACE trial of stent-protected angioplasty versus carotid endarterectomy in symptomatic patients: a randomised non-inferiority trial. Lancet 368:1239-1247

12. Vahedi K, Hofmeijer J et al (2007) Early decompressive surgery in malignant infarction of the middle cerebral artery: a pooled analysis of three randomised controlled trials. Lancet Neurol 6:215-222

13. Jüttler $E$, Unterberg A et al (2014) Hemicraniecto$\mathrm{my}$ in older patients with extensive middle-cerebral-artery stroke. N Engl J Med 370:1091-1100 